\title{
Prevalence of Sinew String in patients coming to a tertiary care hospital of Nepal: A descriptive cross-sectional study
}

\author{
Shrestha $\mathrm{P}^{1}$, Shrestha $\mathrm{K}^{2}$, Acharya $\mathrm{L}^{3}$, Shakya $\mathrm{S}^{4}$ \\ ${ }^{1}$ Associate Professor, and HOD, Department of Prosthodontics, KIST Medical College and Teaching Hospital \\ ${ }^{2}$ Associate Professor, Department of Prosthodontics and Maxillofacial Prosthetics, People's Dental College and Hospital \\ ${ }^{3}$ Lecturer, Department of Prosthodontics, KIST Medical College and Teaching Hospital \\ ${ }^{4}$ Lecturer, Department of Periodontology, KIST Medical College and Teaching Hospital
}

\begin{abstract}
Introduction: Sinew strings are frenum or wrinkle like entity of mandibular distal buccal mucosa which is frequently encountered in the population. Sinew strings play an important role in determining the width and buccal extension of the denture flange of mandibular complete denture.

Materials and Methods: This descriptive cross-sectional study was conducted at a tertiary care hospital from $17^{\text {th }}$ June 2020 to $20^{\text {th }}$ September 2020. Convenient sampling $(n=245)$ was done. Point estimate at $95 \%$ Confidence Interval was calculated along with frequency and proportion for binary data. Data analysis was done in Statistical Package for the Social Sciences version 21.

Results: Among the study population ( $\mathrm{n}=245$ participants), sinew string was seen in $35 \%$ of the participants. The frequency of sinew string present bilaterally was $19 \%$ while it was present unilaterallyin $16 \%$ of the population.

Conclusions: The presence of sinew string in the sampled Nepalese population showed a higher prevalence than previous studies.
\end{abstract}

Key words: Complete lower denture; Denture retention; Denture stability; Sinew string.

\section{Introduction}

$\mathrm{T}$ he sinew string or Someya string is a frenum or wrinkle like entity of buccal mucosa. It is usually found along the distal surface of the lower second molar to the third molar in dentulous mandible, and in edentulous mandible, it is seen from around the anterior border of the retromolar pad as originally described by Seiichiro Someya in $2008 .{ }^{1}$

\section{Conflict of Interest: No}

\section{*Corresponding Author}

Dr. Prabhat Shrestha

Associate Professor, Department of Prosthodontics, Kist Medical College and Teaching Hospital P.O. Box 19444

E-mail: prabhatshrsthadr@gmail.com
Anatomically, there is the presence of pterygomandibular raphe in the deep part where the streaks appear. ${ }^{2}$ When observed macroscopically, the tissue underneath the mucosa of sinew string is composed of collagen fiber bundles, which becomes apparent with association of buccal mucosal movement and tension. ${ }^{1,3}$ The sinew string when present, plays an important role in influencing the width and extension of the mandibular denture in the distal buccal region. Functional movements of improperly designed mandibular complete denture which does not correspond to sinew string's behavior may cause involuntary tension during mastication and swallowing and break the marginal seal, hence reducing the denture's retention and stability. ${ }^{1}$

The sinew string presents itself in diverse anatomical variations and is present in only 
$10-20 \%$ of the population in studies conducted by Someya and Miyake. ${ }^{1,3}$ Due to these reasons, this structure usually goes unnoticed to the general dental practitioner and even to prosthodontists, which may ultimately affect the stability and retention of mandibular removable complete dentures. ${ }^{4}$

There have been very few studies on sinew strings and no study regarding the presence of sinew string in Nepalese population to date. Hence the objective of this study is to study the prevalence of sinew string in Nepalese population coming to the Dental Department of Kist Medical College and Teaching Hospital.

\section{Materials and Methods}

A descriptive cross-sectional study was conducted at KIST Medical College and Teaching hospital from $17^{\text {th }}$ June 2020 to $20^{\text {th }}$ September 2020. Ethical clearance approval was received from the institutional review committee (IRC) of KIST Medical College (reference number:076/077/48) before conducting the study.

A total of 245 (n) no of patients coming to the OPD (outdoor patient department) of Dental Department of Kist Medical College and Teaching Hospital were examined, where,

$\mathrm{n}=\frac{\mathrm{z}^{2} \mathrm{pq}}{\mathrm{d}^{2}}$

$\mathrm{z}=1.96$ and with $95 \%$ confidence interval permissible error of $5 \%$ and prevalence $=20 \%$ from the previous article. ${ }^{1}$

Convenience sampling was used to collect the data from patients visiting the Dental Department of Kist Medical College.

The study included participants above 18yrs of age. Only participants not willing to give consent were excluded from the study.

The side of the mandible having any molar teeth was classified as dentulous while the side missing all the first, second, and third mandibular molars was classified as edentulous, regardless of the presence or absence of other teeth. Hence the right and left side of each participant was classified as either dentulous or edentulous.

After taking written consent, the patients were checked for the presence or absence of sinew string on the right and left side during the following situations

- Just opening of the mouth (if present the sinew string was categorized as clearly visible) Fig.1

- Moving the cheeks sideways with the help of a finger (if present the sinew string was categorized as visible) Fig.2

- Pushing the buccal mucosa on the external oblique ridgewith a finger (if present the sinew string was categorized as slightly visible)

If the sinew string was not seen in the above situations it was categorized as absent.

The data were collected and entered in SPSS version 21 and descriptive statistical analysis (frequency and percentage) was derived.

\section{Results}

Sinew string was present in $35 \%$ of the population among which in $19 \%$ was present bilaterally and in $16 \%$ was presentonly on one side and absent on the contralateral side. Among the 245 participants, 16 participants were completely edentulous on both the right and left sides, and 226 were dentulous on both the right and left sides while 3 participants were dentulous on one side and edentulous on the other side of the mandible. The distribution of sinew strings categorized according to clearly visible, visible, and slightly visible is given in Fig. 3. The distribution of sinew strings on the right and left side with respect to dentulous or edentulous is given in Fig. 4 
Among the 245 participants, 151(61.6\%) were females while 94(38.1\%) were males. The mean age of the participants was $37 \pm 16$ years. The prevalence of sinew string on both sides was

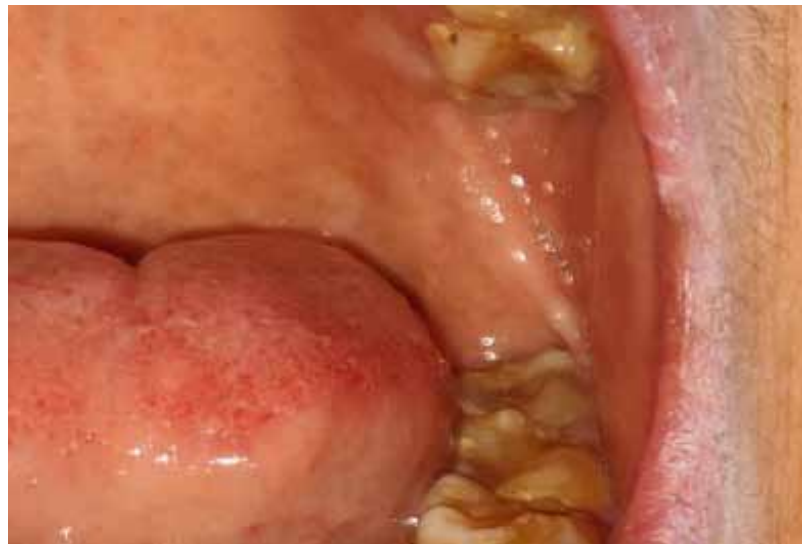

Figure 1: Sinew string seen when just opening of the mouth (sinew string was categorized as clearly visible) similar with respect to gender.The distribution of sinew strings with respect to gender is given in Fig. 5

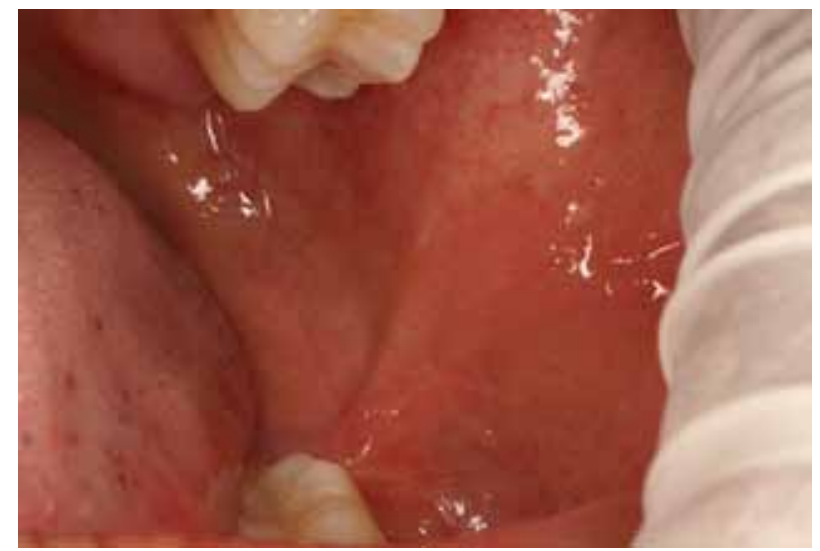

Figure 2: Sinew string seen when moving the cheeks sideways with the help of a finger (sinew string was categorized as visible)

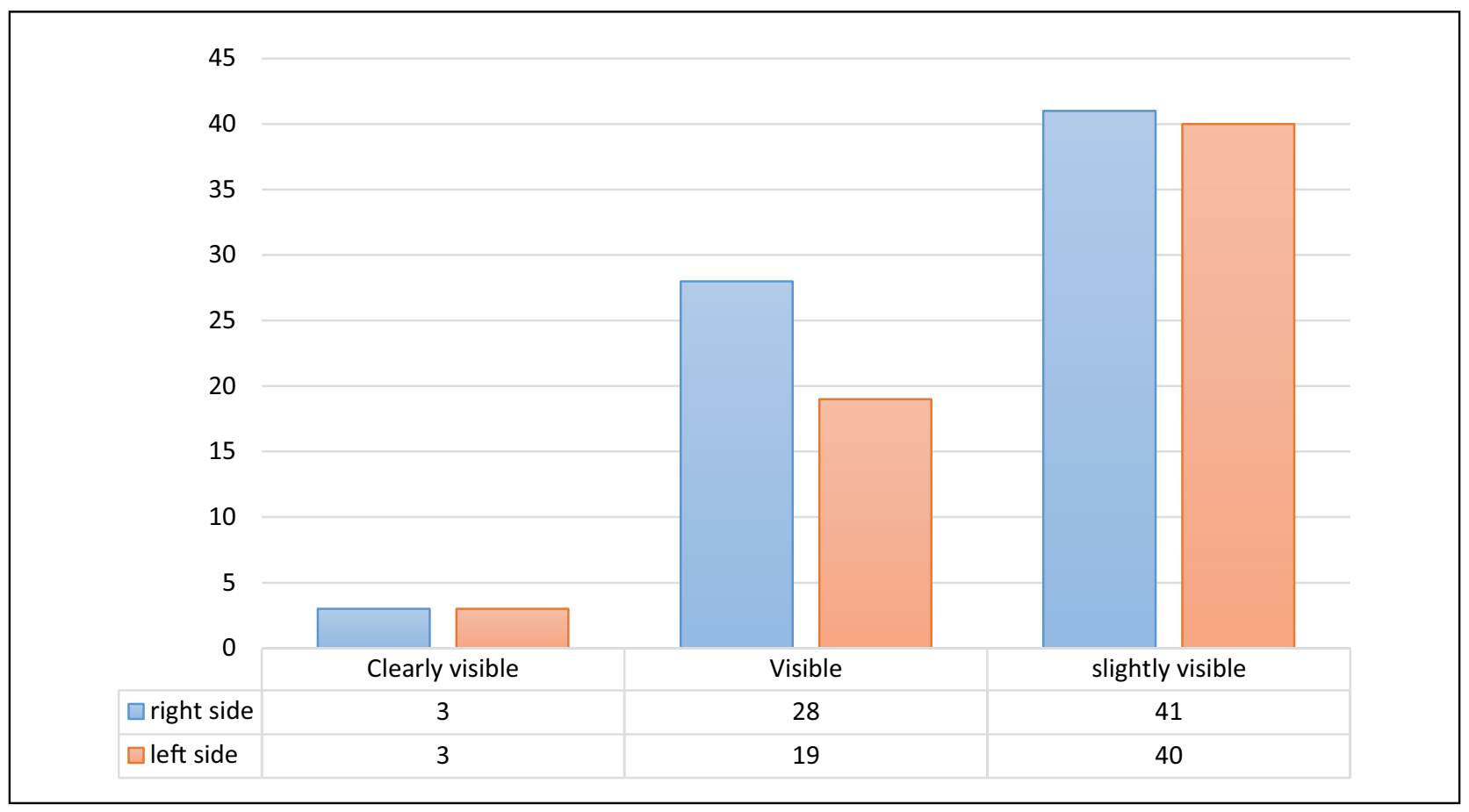

Figure 3: Distribution of categories of sinew string on the right and left side 


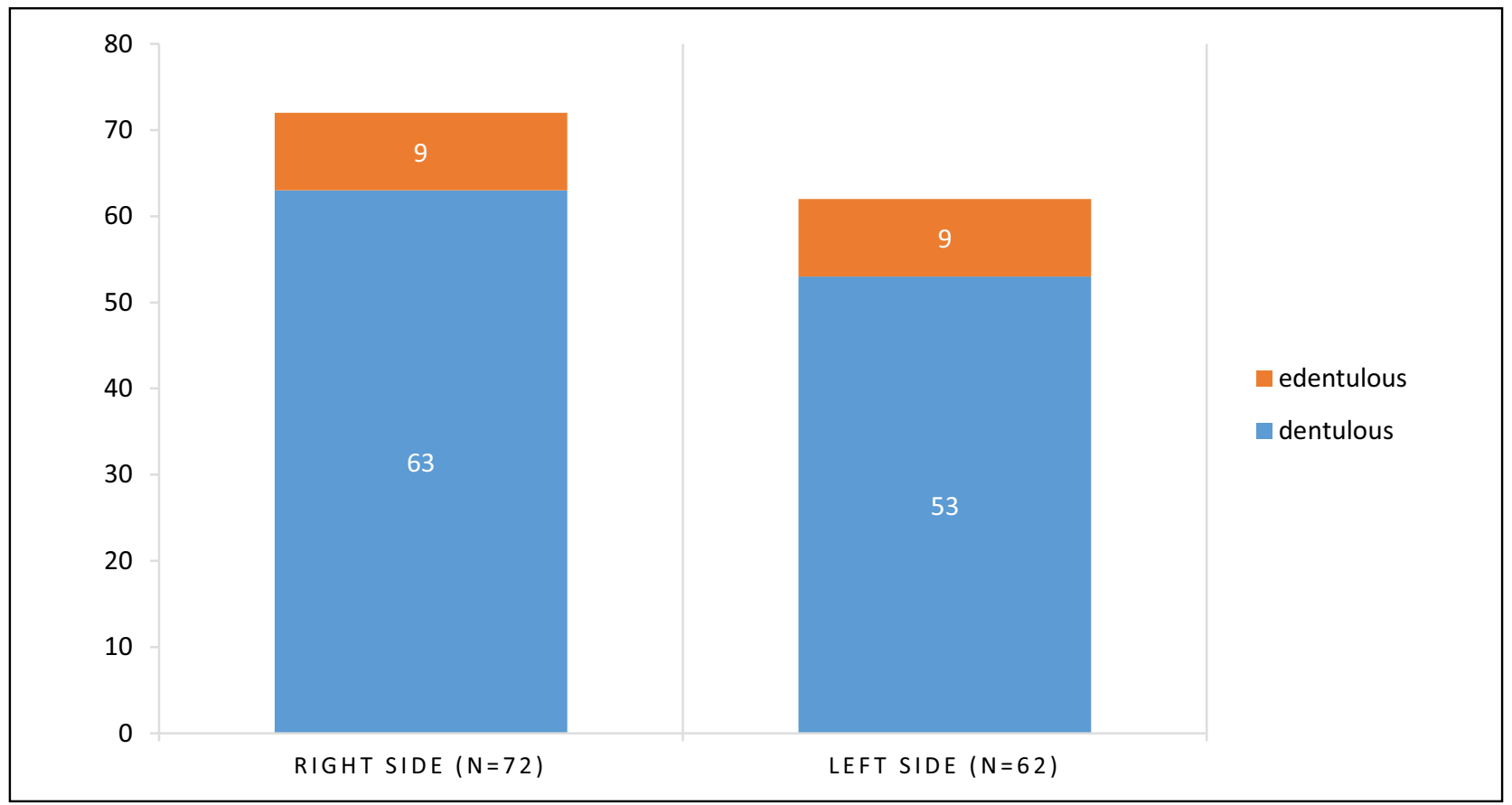

Figure 4: The distribution of sinew strings on the right and left with respect to dentulous or edentulous sides

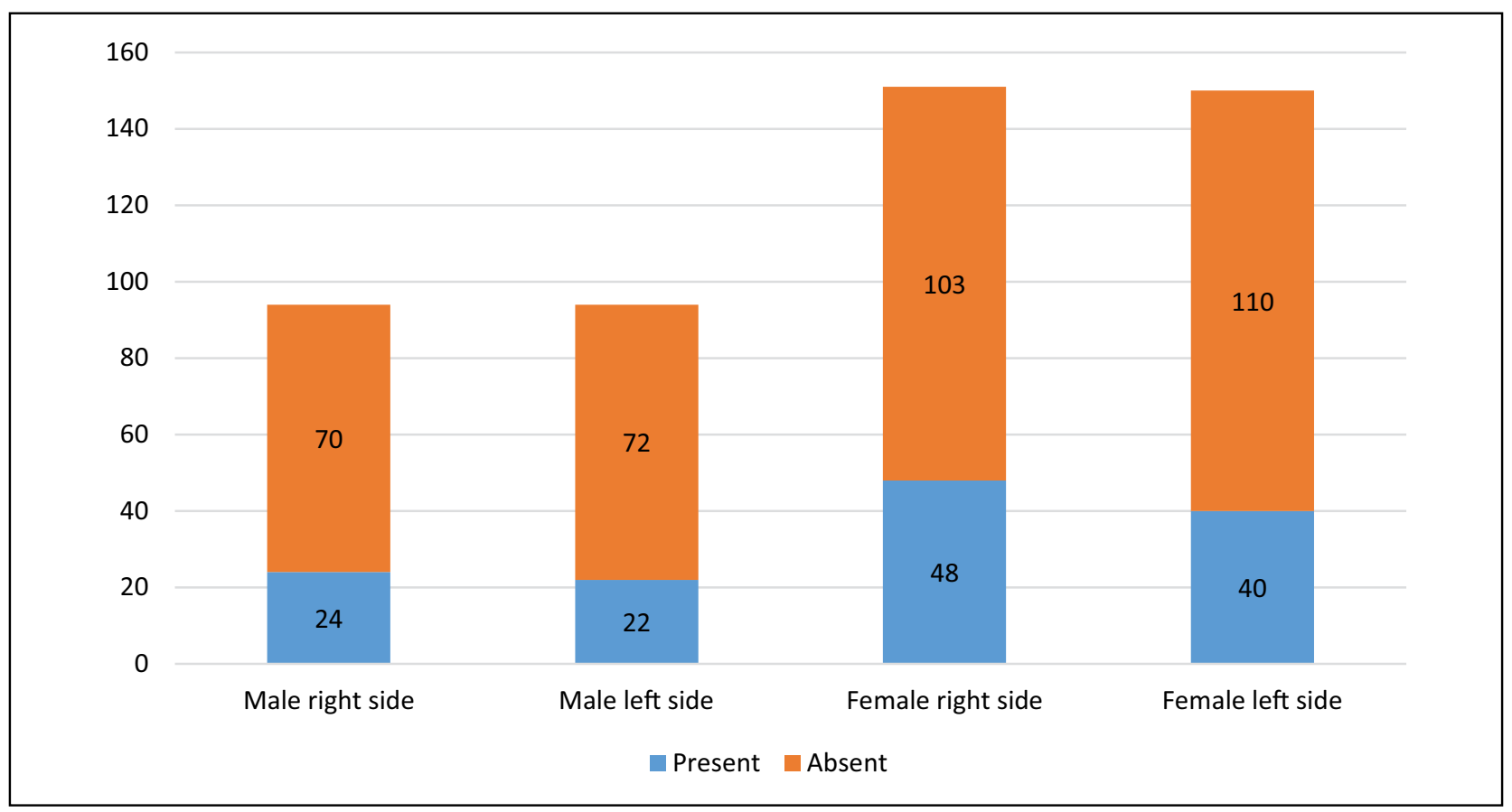

Figure 5: The distribution of sinew strings with respect to gender

\section{Discussion}

The posterior borders of mandibular complete dentures are one of the most difficult areas of obtaining denture peripheral seal so it is necessary to observe this structure before fabrication of the denture. ${ }^{5}$
Seiichiro Someya conducted a study in 2008, assessing the prevalence of sinew string and reported its presence in only $10-20 \%$ of the Japanese population. ${ }^{1}$ Miyake in 2016 conducted a similar study with similar results. Our study however showed that the prevalence of sinew 
string was $35 \%$ in the study population, which is more than the previous studies. Our result emphasizes that a larger study in different populations is necessary to see the variations of this structure.

Categorization of the participant's right and left side as dentulous or edentulous was decided based on the presence or absence of the molar teeth because when the first, second, and third molars are absent the retromolar pads are created. ${ }^{1}$ So even with the presence of other teeth in the mandible, the posterior area will represent an edentulous arch. In our study, among the 245 participants, there were dissimilar numbers of edentulous arch on the two sides.

Unilateral sinew string was found in $16 \%$ of the study population. Findings of the unilateral presence of sinew string were not reported previously and suggests that these structures can be present unilaterally as well.

Somaya reported that there might be more than one sinew string when pushing the external oblique line. ${ }^{1}$ The authors however did not find multiple sinew strings in a single location. This may be due to the low occurrence of sinew string and the percentage of such multiple strings could be even lesser.

Miyake and Abe demonstrated the importance of sinew string in determining the width and extension of denture and described modified impression making techniques to fabricate suction effective dentures in the distobuccal region. ${ }^{3,4}$ It is crucial to examine the presence of this structure as the authors have recognized a higher prevalence rate of sinew strings than previously determinedand modify the impression making techniques for better success of the mandibular denture.

\section{Conclusions}

Sinew strings may be frequently encountered in the distobuccal mandibular region. The knowledge of prevalence and identification of sinew string can play an important role in the proper fabrication of retentive and stable removable prosthesis.

\section{Acknowledgments}

The authors would like to thank Dr. Sijan Poudel for her help in statistical analysis. We are very grateful to Dr. Fumiaki Yamazaki for guiding us and helping in the translation of Japanese articles.

\section{References}

1. Someya S. The Anatomical Study of the Frenum Observed on the Buccal Mucosa of Mandibular Second Molar and Posterior of Retromolar pad. J Clin Dent [Internet]. 2008;28(1-2):14-20. Available from: https://www.jstage.jst.go.jp/ article/jacd1999/28/1-2/28_1-2_14/_article

2. Tsumori N, Abe S, Agematsu H, Hashimoto M, Ide Y. Morphologic characteristics of the superior pharyngeal constrictor muscle in relation to the function during swallowing. Dysphagia [Internet]. 2007 Apr 9;22(2):1229. Available from: http://link.springer. com/10.1007/s00455-006-9063-2

3. MiyakeH. Key points of complete denture 4 "The border shape of Suction Effective Mandibular Complete Denture" (3) "Someya sinew string and Posterior mylohyoid fossa". Dent diamond [Internet]. 2016;41(11):90-6(Japanese). Available from: https://mol.medicalonline.jp/ archive $/$ search $? \mathrm{jo}=\mathrm{aq} 5 \mathrm{dentm} \& \mathrm{ye}=2016 \& \mathrm{vo}=4$ 1 \&issue $=11$

4. Abe J, Kokubo K, Sato K. Mandibular SuctionEffective Denture and BPS: A Complete Guide. Tokyo: Quintessence Pub.; 2012.

5. Someya S. Observing the oral form and denture border form for comfortable border seal. J Pract Prosthodont. 1997;30(1):31-6(Japanese). 